\title{
Entretien
}

\section{Edgard Pisani, croire à la force des idées}

\section{Propos recueillis par Nicole Mathieu, Françoise Plet et Yves Guermond}

\author{
Edgard Pisani ${ }^{\mathrm{a}}$, Nicole Mathieu ${ }^{\mathrm{b}}$, Françoise Plet ${ }^{\mathrm{c}}$, Yves Guermond ${ }^{\mathrm{d}}$ \\ a Ancien ministre du général de Gaulle et de François Mitterand \\ b Géographe, UMR Ladyss, Université de Paris 1, 2 rue Valette, 75005 Paris, France \\ c Géographe, UMR Ladyss, Université de Paris 8, 2 rue de la Liberté, 93526 Saint-Denis cedex, France \\ d Géographe, Université de Rouen, équipe MTG (Modélisation et traitements graphiques en géographie), \\ 76821 Mont-Saint-Aignan cedex, France
}

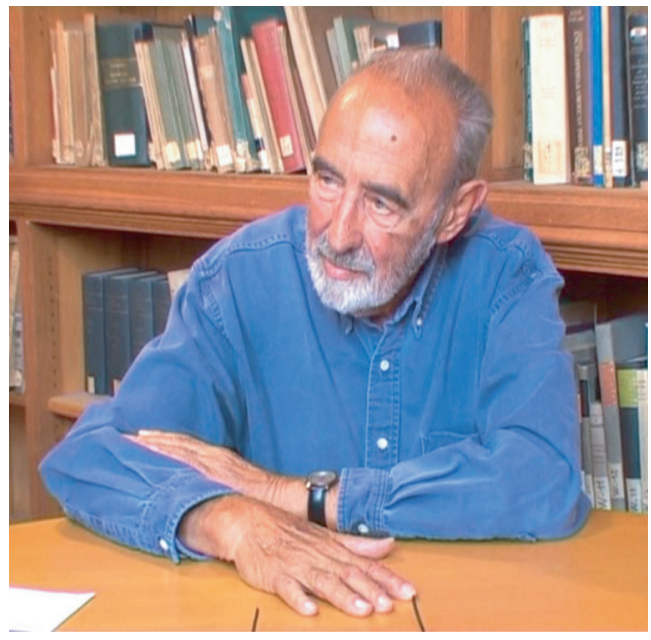

Edgard Pisani : Je souhaite avoir l'intégrale de l'entretien. C'est une manière que j'ai de travailler. Je jette tout ce que j'ai à dire en réponse aux questions que l'on me pose. J'improvise et il y a parfois des formules et des percées que j'aime à voir, parce qu'elles me montrent que j'ai été plus loin que là où j'en étais, et à retravailler.

Nicole Mathieu (NSS) : Comment devient-on Edgard Pisani? Comment expliquer que cet homme, Pisani, qui sous de Gaulle a fait les lois d'orientation agricole de 1960-1962 - tout le monde les appelle la loi Pisani -, puisse, aujourd'hui, alors qu'il est un "vieil homme », nous donner à lire une vision aussi radicale de ce que devrait être le Politique au temps présent? L'origine de

Auteur correspondant : N. Mathieu, mathieu@univ-paris1.fr cette question, certes brutale, est dans notre désir de comprendre, nous les chercheurs, ce qui nous paraît comme un mystérieux passage entre les connaissances et l'action politique, surtout quand elle est juste. N'y a-t-il pas un moment de décalage, une coupure qui s'opère entre les informations dont vous disposez pour « entendre, parler de la réalité et partir des besoins », comme vous l'avez dit d'entrée, et votre capacité à repenser le tout, à revenir sur une position personnelle « et inventer des solutions qu'on ne trouve pas dans les textes »? Comment parvenez-vous à construire cette position éminemment et politique et personnelle?

Edgard Pisani : J'avais 26 ans, et le hasard des choses m'a fait commander à la bataille de Paris, depuis la préfecture de Police, un secteur de Paris. J'ignorais la préfecture, j'ignorais la guerre. J'ignorais tout. J'ai eu à répondre à des besoins qui se sont manifestés, comment dirais-je, en m'exposant en quelque sorte. Je n'ai pas eu l'idée d'aller chercher dans les registres ou dans mon expérience administrative passée la manière d'y répondre, puisque je n'avais pas d'expérience et que l'administration n'en avait pas davantage. Je me suis donc placé tout bonnement comme un simple poilu, comme un simple homme devant une réalité : que faut-il faire là pour s'en sortir? Et ça a marché. Finalement, la préfecture n'a pas été envahie, finalement il n'y a pas eu de morts à la préfecture, finalement le drapeau français est resté sur le toit de la préfecture alors qu'il était menacé de toutes parts.

Je vais prendre un autre exemple. J'étais ministre de l'Agriculture et je résidais beaucoup en Maine-et-Loire où mon fils est viticulteur. J'ai eu à connaître, d'une part, 


\begin{abstract}
Biographie
Né le 9 octobre 1918 à Tunis, Edgard Pisani a fait ses études de droit et de lettres à Paris. Il a débuté sa carrière comme sous-préfet et est devenu, en 1944, chef puis directeur adjoint du cabinet du préfet de police (1945). En 1946, il a été nommé directeur de cabinet du ministre de l'Intérieur, préfet de la Haute-Loire et directeur de cabinet du ministre de la Défense nationale. Il est devenu préfet de la Haute-Marne en 1947, et s'est trouvé en disponibilité à partir de 1953 pour exercer ses fonctions de sénateur de la Haute-Marne (1954-1961 puis 1974-1981), ministre de l'Agriculture (cabinets Michel Debré, 1961-1962, et Georges Pompidou, 1962-1966), ministre de l’Équipement (troisième cabinet Georges Pompidou, 1966-1967), ministre de l'Équipement et du Logement (quatrième cabinet Georges Pompidou, 1967). Il a donné sa démission de membre du gouvernement en avril 1967 pour devenir, entre autres, député de Maine-et-Loire, puis président du Conseil national des économies régionales (1967-1971). Conseiller général du canton de Montreuil-Bellay de 1964 à 1965, il est élu maire de la commune de 1965 à 1975 . Dans les années 1980, il a été membre de la Commission des communautés européennes (à partir de 1981), député du Parlement européen et commissaire européen au Développement. Sous la présidence de François Mitterand, il est délégué du gouvernement en Nouvelle-Calédonie et dépendances (1984-1985), ministre chargé de la Nouvelle-Calédonie en 1985 et chargé de mission auprès du président de la République de 1986 à 1992. Il a été également président du Centre euro-arabe de prospective méditerranéenne, devenu Institut du monde arabe, de 1988 à 1995 et a assuré la présidence du Centre international des hautes études agronomiques méditerranéennes. Il entre au Conseil économique et social en 1992. Auteur de nombreux ouvrages consacrés, entre autres, à l'Europe et au tiers-monde : Défi du monde : campagne d'Europe, Paris, Ramsay, 1979; La Main et l'Outil : le développement du Tiers-monde et l'Europe, Paris, R. Laffont, 1984 ; Pour l'Afrique, Paris, Odile Jacob, 1988; Une autre idée du monde : l'utopie comme méthode, Paris, Le Seuil, 2001; Un vieil homme et la terre : neuf milliards d'êtres à nourrir, la nature et les sociétés rurales à sauvegarder, Paris, Le Seuil, 2004, Edgard Pisani est le fondateur de la revue L'Événement européen dont il a été directeur de publication. Un précédent entretien avait été publié dans Natures Sciences Sociétés : Agriculture et environnement : quelle recherche pour quelle société ? Un entretien avec Edgard Pisani, Natures Sciences Sociétés, 1, 4 (1993), 336-341.
\end{abstract}

le fait que la vallée de l'Authion, qui est une vallée parallèle à la vallée de la Loire, était inondée régulièrement du fait que les levées qui avaient été construites du temps de Colbert empêchaient qu'au moment des crues, l'eau de l'Authion ne se déverse dans la Loire. Ces inondations rituelles rendaient toute agriculture impossible. Se pose donc là un problème d'utilisation de bonnes terres et de régulation de l'eau. D'autre part, et dans le même temps, j'apprenais qu'à Angers se posait un problème, celui de l'urbanisation qui, jour après jour, mois après mois, détruisait des terres horticoles au profit de la construction d'immeubles d'habitation, si bien que l'un des bassins les plus prospères de production de fleurs et d'horticulture en France était en train d'être détruit. De ces deux problèmes qui n'ont rien à faire l'un avec l'autre, on n'en a fait qu'un : si on transposait l'horticulture d'Angers dans le bassin de l'Authion, cela rentabiliserait les travaux nécessaires pour pomper, comme cela se faisait et se fait encore en Hollande, l'eau de l'Authion pour la redéverser dans la Loire. Donc, partir du concret pour résoudre les problèmes. Partir du concret, ce n'est pas seulement l'eau qui monte, c'est aussi les sociétés qui connaissent la misère, c'est tout. Partir de la réalité des problèmes et non pas des institutions. Je crois que c'est une révolution extraordinairement importante à faire en France, que c'est une leçon qui mérite d'être retenue. En France, parce que nous sommes cartésiens, la règle importe plus que le cas. Dans la civilisation anglo-saxonne, parce qu'ils ne sont pas cartésiens, le cas importe plus que la règle. $C^{\prime}$ est donc une mutation fondamentale à opérer dans notre comportement, dans nos procédures : non pas de quelle loi s'agit-il et comment la mettre en œuvre, mais de quelle réalité s'agit-il et comment y répondre? Voilà la leçon fondamentale de tout, presque tout, presque pratiquement toute ma vie.

J'ai été médiateur dans la guerre civile qui déchirait le Mali il y a quelque 15 ans et je me suis trouvé en face de populations sédentaires noires, les Bambaras et les Songhai, et d'une population nomade de Touaregs. On m'avait demandé de venir au Mali pour conseiller le gouvernement dans sa politique agricole. À la suite d'une conversation que j'ai eue, par hasard, à ce sujet avec le Premier ministre, celui-ci est allé trouver le président en lui disant: «Monsieur Pisani, il ne faut pas l'utiliser pour s'occuper de l'agriculture, on en trouvera d'autres, car lui, il sait parler des problèmes tels qu'ils sont, confiezlui le dossier de la médiation entre le gouvernement de Bamako et les Touaregs. » Et je suis allé de place en place à Bamako, puis dans le désert. .. Et par quoi ai-je commencé? J'ai commencé par me taire. Je ne savais rien, il fallait que j'apprenne. Et, ce qui a le plus impressionné les gens, c'est que je ne leur ai pas dit, avant de les entendre, ce qu'il fallait qu'ils fassent, mais je leur ai demandé de me dire, avant que je ne parle, les problèmes qui se posaient à eux. J'évoque une conversation extraordinaire que j'ai eue avec le prince des Touaregs, l'aménokal (ce mot désigne le chef de tribu en tamacheq); elle a duré de l'ordre de trois heures et nous avons peut-être échangé en trois heures cinq mille mots, c'est-à-dire à peu près autant que ce que nous avons fait à l'instant en quelques minutes. Le lendemain, le prince $\mathrm{m}^{\prime} \mathrm{a}$ fait dire par son fils qu'il acceptait de négocier avec moi parce que j'avais su écouter. C'est donc aussi, au-delà de la logique même qui consiste à partir des besoins, la logique qui consiste à écouter ceux qui ont un problème et qui, parce qu'on les écoute, prennent confiance dans la démarche qui est 
entreprise vers eux. C'est donc bien une philosophie, une manière de construire l'action politique : parler moins et écouter davantage, et, à partir de là, construire - alors là, dans un jeu dialectique entre ce qu'ils vous disent et ce que vous avez en tête, entre ce que les uns vous disent et ce que vous avez en tête, ce que les autres vous disent et ce que vous avez en tête, et entre les uns et les autres -, construire progressivement un système dialectique qui conduit à la médiation. Un dernier mot : je m'amuse à écrire un glossaire sur les mots singuliers que je rencontre, parce que cela aide à la réflexion ensuite. Dans le glossaire, il y a les mots arbitrage et médiation : l'arbitre coupe la poire en deux, le médiateur donne aux adversaires les raisons de travailler ensemble. Et, au fond, j'ai donné au Touaregs et aux Noirs maliens les raisons de travailler ensemble. Vous en savez, je crois, assez sur le bonhomme.

Yves Guermond : En ce qui concerne l'Europe, vous dites dans votre livre que ce qui lui manque, c'est une volonté politique. Vous y écrivez «que le système institutionnel européen actuel ne favorise pas les solutions » et, à un autre moment, "qu'il faudrait vendre les productions européennes à un prix correspondant à leur coût ». Comment voyez-vous l'avenir de la politique européenne?

Edgard Pisani : Je considère que l'élargissement est une monstruosité. Vu mes idées, je l'accepte sauf à nier l'Europe. J'ai donc le choix entre accepter une difficulté énorme et travailler avec pour essayer de la surmonter, ou nier ce à quoi j'ai cru depuis 50 ans. J'ai choisi de continuer à travailler sur ce à quoi j'ai cru depuis 50 ans. Quelle est la raison fondamentale pour laquelle je suis contre les élargissements et voudrais trouver une solution (j'y reviendrai dans un instant)? La réponse est dans l'analyse des raisons fondamentales qui font de la construction européenne un acte historique majeur. Je crois que nous assistons à la fin d'un rêve qui a été esquissé du temps de Truman et qui a progressivement pris corps jusqu'à Clinton - Clinton compris -, c'est celui de l'empire américain. L'empire américain était un système unilatéral de consultation marginale qui s'est fait au détriment d'un système multilatéral, d'un système vrai de concertation entre acteurs différents aux intérêts parfois contradictoires. Quand je me suis trouvé devant cette réflexion, je me suis alors heurté à un vrai problème : est-ce que l'on peut passer de l'empire américain, singulier, et quasiment exclusif, à 250 unités dont certaines ne comprennent que 500000 personnes? Et, progressivement, j'en suis arrivé à la conception d'un monde qui serait dirigé - dirigé, le mot est trop fort -, organisé, guidé, animé par deux conseils - et c'est une innovation que j'ajoute à mon raisonnement - : le Conseil de sécurité, puisqu'il existe, et le Conseil de développement, puisqu'il faut le créer. Je l'ai écrit dans mon livre précédent, L'Utopie comme méthode ${ }^{1}$, et j'en suis arrivé à dire qu'en fait, il n'y avait que cinq réalités politiques objectivement capables de jouer un rôle directeur dans l'animation et ce que j'ai appelé tout à l'heure l'organisation du monde : la Chine, l'Inde, la Russie, l'Europe et les États-Unis. À eux cinq, ils représentent de l'ordre de $75 \%$ de la population du monde, un peu moins peut-être, et 90 à $92 \%$ de la richesse mondiale. Si bien que j'ai imaginé dans mon livre précédent, je le répète, l'idée d'un système international avec deux niveaux : un niveau de délibération et d'orientation qui est l'assemblée générale des nations du monde, ce qui existe ; et un système organisationnel et exécutif en même temps, qui est au fond le G7 institutionnalisé au terme du système des Nations unies, ce G7 se divisant lui-même en deux, la branche développement et la branche sécurité. Je crois que nous ne passerons pas au travers de cette réforme. Cela prendra le temps qu'il faudra, mais il est clair qu'on l'on ne pourra pas très longtemps imaginer que la France et l'Angleterre représentent l'Europe. Il faudra que l'Europe se représente elle-même. On ne pourra pas imaginer que l'Inde ne fasse pas partie du Conseil de sécurité. Donc, dans l'utopie - et c'est une des choses que je veux souligner -, dans les utopies que j'articule successivement, le réel prend la place essentielle. C'est un réel réorganisé mentalement, revu contre les institutions, ce n'est pas quelque chose qui fasse fi du réel. Le réel, c'est qu'il y a cinq entités dans le monde qui représentent puissances, populations et richesses et qu'il leur revient d'exercer les principales responsabilités du monde.

Françoise Plet : Pour ce qui concerne les responsabilités en matière d'alimentation, vous insistez, à un moment du livre, sur la liaison qu'il y a entre disponibilité alimentaire, de qualité et saine, disponibilité en eau, de qualité et saine, éducation et système sanitaire. Et ces trois ou quatre choses sont pour vous particulièrement reliées. Or, dans le monde d'aujourd'hui, dans les organisations internationales, ces éléments sont gérés et financés de manière thématique, finalement : il y a une organisation pour l'alimentation, une organisation pour la santé, une organisation pour les épizooties animales, une organisation pour l'éducation, une organisation pour l'enfance...

Edgard Pisani : Arrêtez, arrêtez, je connais la liste...

$$
\text { F.P. : Alors oui, justement, comment faire? }
$$

Edgard Pisani : Je prendrai un exemple qui est très frappant, si vous voulez, c'est celui de l'Organisation mondiale de l'agriculture et de l'alimentation, dite FAO. Il est clair que la FAO représente un des éléments essentiels de la régulation mondiale, à cause de la faim dans le monde, à cause d'un tas de choses... Il est clair en même temps qu'on lui a enlevé tout pouvoir en créant le Conseil mondial de l'alimentation, etc. C'est-à-dire qu'on a mis

\footnotetext{
1 Pisani, E., 2001. Une autre idée du monde : l'utopie comme méthode, Paris, Le Seuil.
} 
en place des institutions pour qu'elles ne servent à rien ; et, quand elles servent à quelque chose, c'est marginal et, après tout, tel ou tel pays dans le monde peut refuser d'y adhérer. Alors, première réponse à la question que vous me posez, il est clair que le système international est divisé en deux grands ensembles : un grand ensemble relevant du système des Nations unies, qui est en nombre et en volume le plus important, et les ensembles collatéraux, qui sont beaucoup moins importants en volume et qui, finalement, jouent le rôle principal - le Fonds monétaire international et la Banque mondiale. Donc, nous n'avons pas un système mondial cohérent, nous avons un système mondial fractionné entre zones, où seuls les grands sont assis à la table. Et, problématique générale, nous avons, à l'intérieur du système des Nations unies, un nombre incroyable d'agences dont chacun des directeurs généraux se prend pour le grand Mamamouchi. Donc, une réforme fondamentale des Nations unies s'impose autour de deux conseils : un Conseil de sécurité, un Conseil de développement, et d'un système d'agences dont les actions sont elles-mêmes soumises à des disciplines par rapport au système d'ensemble. Je me rappelle des délibérations du Conseil de l'alimentation, nous nous foutions de ce que pouvaient dire les Nations unies, de ce que pouvait dire la Banque mondiale, etc., nous avions notre propre politique. À l'intérieur de la Communauté européenne, puisqu'elle s'appelait comme cela de mon temps, le Conseil des ministres de l'Agriculture négociait à sa manière avant d'en référer au système central qui était le Conseil européen. On a donc bureaucratisé les fonctions en leur donnant des pouvoirs bureaucratiques, elles ont cru en faire des pouvoirs politiques, elles ont cru en faire des pouvoirs exécutifs, et le résultat est que la totalité du système est paralysée. Regardez le pauvre monsieur Koffi Anan, qui appelle au secours au profit du Soudan et n'arrive à rien obtenir! Regardez ce qui se passe dans l'environnement, il prêche dans le désert, c'est le cas de le dire, parce que les États-Unis ne marchent pas. Nous n'avons pas un système cohérent qui soit tel que, une fois qu'une décision est prise, chacun soit dans les faits amené irréductiblement à y adhérer. Enfin, j'ai oublié de citer, parmi les agences qui échappent aux Nations unies, celle qui joue actuellement le plus grand rôle : l'Organisation mondiale du commerce.

Je réponds maintenant à votre question, monsieur Guermond : on ne peut pas attendre des systèmes qui sont en place qu'ils s'autoréforment. Car la fonction fondamentale des institutions, c'est de persévérer dans le temps. Si on ne projette pas, d'une certaine façon, une vision du monde qui, à la fois, démontre l'inanité de ce qui existe et l'utilité de ce qui pourrait exister, on n'a aucune chance d'avancer. Je vais vous citer un exemple qui me vient à l'esprit et qui est un exemple concret, historique, je dirais presque. Dans une institution française chargée des études stratégiques internationales, un des cadres de cette institution recevait un jour une délégation de très haut niveau originaire de Chine. La conversation en vient à la problématique des institutions internationales. Cette personne, que je ne connaissais pas, que je connais depuis, avait sur sa table mon livre L'Utopie comme méthode. Il a dit aux Chinois : "Voilà l'idée qui vient d'être émise par monsieur Pisani : qu'est-ce que vous en pensez ?» Réponse des Chinois : "C'est cela qu'il faut faire et cela se fera. » Je veux dire, donc, que peut-être - pour répondre à votre question de tout à l'heure, Nicole Mathieu -, peutêtre que la différence profonde que j'ai avec la majorité de ceux qui s'occupent de politique, c'est que je crois à la force des idées.

F.P. : Et ce système que vous imaginez, l'utopie que vous imaginez, comment peut-elle concrètement arriver à faire...

Edgard Pisani (en tapant vigoureusement sur la table) : Ce n'est pas mon boulot!

\section{F.P. (rires) : Voilà ! D'accord !}

Edgard Pisani : Ce n'est pas mon boulot! Alors là, il faut être clair. Il y en a dont le métier est d'essayer d'inventer, de dire pourquoi et globalement comment, et non pas de mettre en place des boulons et des rivets! C'est à d'autres de faire ce boulot. Ce n'est pas à moi. Je ne peux pas être en même temps celui qui essaie d'imaginer ce qui est possible, non, plus exactement, celui qui essaie de préciser, d'analyser ce qui est nécessaire et, à partir de là, d'imaginer ce qui est possible... et de laisser un message... Je ne peux pas faire davantage! Je l'ai fait quand j'étais au ministère de l'Agriculture, où je suis parti d'une utopie considérée comme hypothèse et je l'ai réalisée, d'un bout à l'autre, quitte à me tromper sur certains points.

Aujourd'hui, un problème s'impose à ma réflexion : le premier besoin, le besoin fondamental étant l'alimentation suffisante pour la totalité des humains, est-ce que nous sommes sûrs d'alimenter les neuf milliards d'humains en perspective? Ma réponse est que ce n'est pas exclu, mais que ce n'est pas assuré non plus. Je peux entrer dans le détail, mais je le ferai très schématiquement pour l'instant. J'ai essayé de mettre en place les facteurs positifs et les facteurs négatifs. Les facteurs positifs, c'est la science, ce sont les OGM, ce sont de possibles économies dans la consommation; ça, on voit très bien comment faire. Les facteurs négatifs, c'est que l'eau est en train de manquer, $c^{\prime}$ est que le niveau des mers est en train de monter, c'est que le climat est en train d'évoluer, c'est qu'on ne voit pas comment nourrir ceux qui n'ont pas de ressources autres que leur propre agriculture. Ma conclusion est que le monde a besoin de toutes les agricultures du monde. Il faut non seulement favoriser ceux qui peuvent produire plus et exporter, mais favoriser ceux qui, actuellement, n'important pas, ne peuvent pas se nourrir. 
Je crois que ce serait un crime contre les pauvres de ne pas favoriser le développement de leur propre agriculture. Et je crois que c'est une opération scandaleusement dangereuse que celle à laquelle on se livre maintenant, et je vais l'analyser. Cette opération consiste à dire : les États-Unis et l'Europe vont cesser les subventions à leur agriculture, ce qui va donner un avantage à l'Argentine, à l'Australie, au Canada, à la Nouvelle-Zélande, mais aussi à la Russie, à l'Ukraine, à la Pologne, à la Roumanie et à la Bulgarie, qui, les uns ou les autres, bénéficient de conditions climatiques, de niveaux de salaires ou de situations foncières qui leur permettent de produire meilleur marché. On est en train de mettre en place un système dans lequel sept, huit ou dix pays dans le monde vont pouvoir pendant un temps nourrir la totalité du monde et ruiner, grâce au marché, ceux qui n'auront pas la capacité de produire aussi bon marché, et nous nous trouverons bientôt devant un monde dominé par une espèce de quasi-monopole - les pays du groupe de Cairns, pour l'appeler par son nom -, qui pourra profiter de prix de plus en plus élevés, accablant de plus en plus ceux dont nous prétendions nous occuper au départ, c'est-à-dire ceux qui ont faim. Nous sommes entrés dans une logique absolument monstrueuse et le problème, c'est de démontrer, en croyant à la force des idées, en croyant à la force des analyses, c'est de démontrer que la voie dans laquelle on s'est engagé ne peut que détruire le droit des peuples à se nourrir eux-mêmes, comme l'a dit Bertrand Hervieu. En détruisant ce système, on aboutit à un grand désordre et sans doute à l'accablement des plus pauvres. Voilà mon raisonnement. Il est, je crois, assez clair.

F.P. : Est-ce que ce peut être une logique d'État ou, au contraire, - en géographe on peut se poser la question infra-étatique ou supra-étatique?

Edgard Pisani : Je ne crois pas du tout qu'il faille laisser à chaque État, à chaque ensemble, puisque l'Europe n'est pas un État, le droit de définir comme il le veut l'organisation dont il se dote. Mais je crois qu'il faut reconnaître, dans le cadre d'un code de conduite très général, la possibilité pour les pays d'assurer leur sécurité alimentaire, à la condition que la sécurité qu'ils s'assurent ne soit pas un élément de perturbation des marchés mondiaux. Attention, il faut bien prendre le schéma que je présente comme un ensemble qui comporte deux choses : il comporte la protection des marchés européens et américains en particulier, mais l'interdiction d'aider d'aucune façon des exportations. Et donc, le prix garanti n'est pas un prix de faveur, c'est un prix situé au niveau du prix de revient intérieur, c'est-à-dire qu'il n'y a pas là de quoi favoriser une plus-value interne des productions alimentaires, plus-value grâce à laquelle on exporte à bas prix, mais simplement de trouver un équilibre. Je ne dis pas que c'est facile, mais il y a eu des expériences dans l'histoire, je n'y reviendrai pas parce que je les connais mal au demeurant, il y a eu l'Office du blé en France en 1936, il y a eu le système exactement inverse, celui des Deficiency payments en Angleterre, ce sont des systèmes qui ont fonctionné, on sait faire cela, pourquoi ne le fait-on pas? Et si on ne le fait pas, qu' on me dise comment les pays en voie de développement, comment les pays pauvres pourront assurer leur alimentation. Dans les pays en déficit alimentaire, je crois qu'il faut favoriser à toute force l'agriculture, et - je l'ai ajouté dans le livre je crois que l'une des manières $d^{\prime} y$ favoriser l'agriculture, c'est de leur donner une aide alimentaire, mais avec interdiction qu'ils la distribuent gratuitement, de façon qu'ils constituent eux-mêmes, grâce à cette alimentation que nous leur avons donnée, et aux ressources qu'elle procure, un mécanisme de développement. Il faut générer, non pas par un apport en argent, mais par un apport en nature, une dynamique de développement, de façon que l'agriculture se développe.

Y.G. : Mais est-ce que ce sera un appui pour la modernisation de cette agriculture?

Edgard Pisani: C'est le seul chemin de modernisation de l'agriculture : c'est qu'il faut leur donner à manger. Je vais vous citer un exemple. Il y a trente ans environ, monsieur Houphouët-Boigny étant le président de cette République, la Côte d'Ivoire fut subventionnée pour sa production en riz. Les producteurs ivoiriens étaient donc rémunérés à un prix plus élevé que celui du blé mondial qui aurait pu être acheté par le gouvernement ivoirien. La décision est alors prise d'annuler la garantie donnée aux producteurs ivoiriens et donc d'importer du riz, en particulier d'Asie. Le résultat, c'est qu'au bout de cinq ans, la Côte d'Ivoire est devenue déficitaire en riz et qu'actuellement, elle importe plusieurs centaines de milliers de tonnes, peut-être même de millions de tonnes de riz.

Je suis long, mais je veux prendre un autre exemple. J'ai été un jour envoyé par la FAO à Rome, comme président de commission d'enquête, en Égypte qui souffrait de désarroi agricole, malgré la vallée du Nil, malgré le barrage d'Assouan. Nous étions sept : un Américain, un Néerlandais, un Italien, un Indien, un Maltais, un Français... Nous allons nous promener et nous constatons que l'un des malheurs de l'Égypte, c'était que, n'ayant plus à attendre la montée du Nil, les fellahs, les fermiers, avaient acheté des pompes, pompaient dans le Nil, qui restait à niveau constant, pour irriguer leur champ ; mais ils surarrosaient si bien que l'eau descendait dans les profondeurs du sol, rencontrait la nappe saline et que le sel remontait, il y avait une salinisation. Nous en sommes arrivés à une première conclusion. Avec cette première conclusion, je suis allé voir le ministre de l'Agriculture en disant : « Il faut empêcher la surirrigation, sans cela vous n'aurez bientôt plus de terres. » Ce qui est maintenant le cas d'ailleurs. «Et comment faire? - Eh bien, faites payer l'eau. - Ah, non! On ne fait pas payer Dieu, le Nil est Dieu 
pour nous. - Alors le Nil est un dieu bien malfaisant, ce n'est d'ailleurs pas lui qui est malfaisant, mais l'usage que les fellahs en font. - Non! Non! On ne fera pas payer l'eau. » Mais ils font payer l'eau maintenant. Et puis dans la deuxième partie de notre enquête - je continue mon récit -, nous essayons de voir dans quelle mesure il est possible de créer une agriculture qui s'organise pour faire face aux besoins du pays. Nous arrivons à la conclusion qu'il faut faire des ensembles régionaux d'unités de production, autonomes mais complémentaires les unes des autres, et organisées de telle sorte qu'elles soient complémentaires : qu'il y ait une sucrerie pour transformer la canne à sucre ; qu'à partir des déchets de cette fabrique, l'on fasse des aliments du bétail; qu'avec les aliments du bétail, on élève des bovins ou des caprins; qu'avec cet élevage, on crée des fumures possibles, etc. Et donc qu'on recrée, volontairement, ce que l'histoire a créé au cours des siècles dans nos civilisations. Question du ministre, toujours la même : «Est-ce que nous produirons moins cher que ce que nous achetons sur le marché mondial? » Réponse : "Non, parce que vous allez avoir à payer l'investissement initial de votre démarrage. Mais, progressivement, peut-être que oui. De surcroît, il vaut mieux que vous soyez autonourris plutôt que d'importer de quoi vous nourrir, plutôt que d'acheter des machines pour vous développer. » Ils n'ont jamais voulu faire cela.

Donc, il y a deux logiques qui se font front et qui sont profondément contradictoires : d'un côté, la logique de la globalisation, le marché, où la création de produits quels que soient les lieux où ils sont créés - résout tous les problèmes du monde, et de l'autre, celle du territoire, où c'est à partir de ce qui existe que doit naître l'équilibre économique. Ces deux logiques s'opposent et c'est maintenant le moment de poser très clairement le problème. Ce qui me frappe, actuellement, c'est la résignation des organisations professionnelles agricoles européennes et françaises qui considèrent comme inéluctable la mondialisation, la suppression des aides et donc le déclin définitif de l'agriculture. Ne croyez pas que cette position soit une position strictement française, c'est aussi une position américaine. . J Je vis six mois de mon année au ÉtatsUnis, où il y a maintenant une montée des angoisses, des inquiétudes et de la révolte des agriculteurs contre une pratique qui aboutira à leur disparition. Quand on paye, pour une heure, en France, aux États-Unis, ce qu'un ouvrier agricole argentin reçoit pour un jour, il est difficile d'être compétitif.

Alors j'en viens à mon développement de tout à l'heure sur la modernisation agricole. J'ai vécu tout un tas de choses en Afrique où j'ai passé quatre années de ma vie. Le hasard m'a fait visiter une bananeraie, puis une autre, puis une troisième ; mais, en allant au bord de cette dernière, je découvre à côté de la bananeraie une brousse brune, noire. J'ai alors demandé au patron qu'il me permette d'emprunter son hélicoptère pour survoler la bananeraie. J'ai vu la superficie bananière, et autour rien, la mort, rien. C'est-à-dire que la création d'une bananeraie prospère, très prospère, dans une région où il $\mathrm{y}$ a de bonnes terres et où il y a l'eau, n'entraîne d'aucune façon le développement paysan alentour. On se trouve devant ces réalités. Et il faut essayer de voir comment repartir. On ne peut pas dire aux gens : «Partez de rien pour... » Il faut leur dire : «Ce que vous produirez sera payé à un prix qui vous convient et, dans l'attente du jour où vous en arriverez à ce résultat, vous recevrez une alimentation dans les conditions qui vous permettent de survivre. »

N.M. : Est-ce que ce raisonnement sur la manière de construire une position politique juste ne repose pas sur deux choses qui sont quand même hypothétiques ? D'une part, il faut, comme vous le dites, connaître la réalité, ou tout au moins écouter les gens pour la connaître et pour, selon vous, aller plus loin jusqu'à énoncer la solution. Mais, d'autre part, la plupart des exemples que vous nous avez donnés sont d'autant plus convaincants que les réalités sont passées par vos propres yeux, par votre propre expérience. Or, quand on vous lit - et il s'agit d'aspects qui concernent ce que vous dites de la ruralité, du rapport ville/campagne... -, j'ai le sentiment que vous n'êtes pas toujours aussi convaincant; comme si, dans ce cas, vous étiez passé beaucoup plus par la lecture de textes, l'écoute d'informateurs ou de chercheurs privilégiés plutôt que par votre propre expérience...

Edgard Pisani : Moi, je ne suis pas agriculteur d'Afrique.

N.M. : Non, non! Je ne parle pas d'Afrique et je revenais à l'Europe, voire même à la situation française, pour transposer votre raisonnement à la réalité de nos sociétés rurales, urbaines postindustrielles et à une relation qui, pour nous, se décline en termes d'agricultures/villes/ruralités/natures. Votre raisonnement s'appuie en quelque sorte sur deux piliers : d'une part, prendre en compte la réalité au plus précis, au plus divers et au plus exact possible ; d'autre part, avoir la capacité de reformuler les problèmes et, en particulier, de formuler l'importance qu'il faut accorder aujourd'hui à la question de la nourriture. Mais comment prendre en compte la réalité ici, alors que ce ne sont pas forcément les citadins qui sont les plus consommateurs et que les sociétés rurales et urbaines sont devenues si complexes? Où trouver, ici, la capacité de reformuler le problème au niveau même où vous le posez, celui de nourrir le monde? Comment, ici, lie-t-on connaissances et expérience, mise au jour des réalités et portée de l'action politique? Cela suppose de n'être pas seul, cela suppose d'autres gens comme vous!

Edgard Pisani : Eh bien, la politique, c'est quoi alors? Faire des lois, faire la guerre et laisser les gens crever de faim? 
N.M. : Non! Justement!

Edgard Pisani : Alors il faudra bien s'en sortir.

N.M. : En effet!

Edgard Pisani : Ce que je propose, ce n'est pas la manière politique d'en sortir, ce sont les perspectives qui justifient que l'on en sorte, et les voies et moyens par lesquels on peut, après débat, y parvenir. Après débat, parce que je ne suis pas sûr que j'aie raison sur le détail des propositions que je fais. Je suis sûr des objectifs, je suis sûr de la nécessité de le faire et sûr des esquisses que je propose comme solution à la situation présente. Je regrette que notre débat intervienne trop tôt d'une certaine façon. J'ai été invité à un débat public en octobre par le ministre hollandais de l'Agriculture, président du Conseil des ministres de l'Agriculture européens. Dans ce débat public à Amsterdam, tout cela va être discuté à fond. Il va me dire que rien n'est possible; et je lui répondrai que je ne sais pas si c'est parce qu'ils sont abrutis par l'administration ou si c'est parce que ce n'est pas possible. Je lui demanderai comment font les gens des polders pour produire aussi bon marché que dans les régions de l'Argentine où la terre et la main d'œuvre ne valent rien, alors que le polder vaut cher à l'hectare et que le travailleur agricole hollandais vaut quelque chose.

Y.G. : J'étais un peu sceptique par une de vos une phrases, dans laquelle vous parlez de promouvoir une civilisation rurale que vous pensez distincte de celle des villes. C'est un propos qui me choque un peu.

Edgard Pisani : Ce que je constate, monsieur, c'est qu'est en train de se développer dans la région parisienne, dans la région de Toulouse, en Normandie, dans le Nord, en Alsace, une civilisation rurale qui est dominée par le citadin qui a acheté une ferme et qui s'y installe le samedi et le dimanche. La vie rurale est maintenant rythmée, dans un nombre considérable de communes, par la vieille maison que des Hollandais, des Allemands, des Anglais, des Italiens, des Parisiens... ont retapée. On est en train de voir une civilisation qui se dégrade sous nos yeux. Parallèlement, dans le nord de la Bretagne, dans le nord du département des Côtes-d'Armor, une expérience est faite dont l'animateur s'appelle Pochon ${ }^{2}$, qui, lui, arrive à vivre bien avec 40 hectares, et elles sont environ 300 fermes à suivre les règles du jeu. C'est le premier problème qu'il faut donc analyser très à fond - et je vais en Bretagne passer plusieurs jours pour essayer de comprendre dans le détail ce qui se passe, comment cela se passe, pourquoi cela se passe là et pourquoi pas ailleurs. Étant entendu qu'il faut que je me méfie là du problème des lisiers et de l'élevage intensif de porcs qui aboutit à la dénaturation des nappes phréatiques et donc à la destruction de l'environnement.

${ }^{2}$ Formant le réseau d'agriculture durable (Ndlr).
Le deuxième problème est né de l'expérience que j'ai faite étant maire de la commune de Montreuil-Bellay, puisque j'avais ma maison non loin de là. Je présidais le bureau électoral et, comme je n'avais pas à rentrer chez moi - c'était trop loin -, j'ai demandé à mes adjoints de rester avec moi pour regarder la liste électorale. Et nous avons regardé la liste électorale nom par nom. Montreuil-Bellay, qui était une commune de 1500 habitants, a maintenant 5000 habitants grâce aux industries qui s'y sont implantées. Bon! Qu'avons-nous constaté? D'une part, qu'il n'y avait pratiquement pas de fermes dans Montreuil-Bellay, pratiquement pas, peut-être trois ou quatre; d'autre part, que la moitié de la population qui habitait à Montreuil-Bellay n'était pas née à MontreuilBellay. Troisièmement, en réfléchissant sur ces données, que le pays rural autour avait besoin de Montreuil-Bellay pour tout ce qui n'était pas l'acte de produire lui-même, ou l'acte de vivre lui-même. Ainsi donc, nous avons un espace d'activités et de survie, isolé des réseaux de satisfaction des besoins, et un système de réseau de satisfaction des besoins qui ramène tout à la ville. Le lieu où il $\mathrm{y}$ a le Crédit agricole, l'atelier de réparation des machines agricoles, le collège agricole, etc., n'abrite pas le conseil municipal, n'a aucun paysan en son sein, et les paysans qui sont tout autour ne participent pas à la gestion de l'espace dans lequel leur destin se joue, c'està-dire la commune. Alors j'en arrive à me demander : Est-ce que nous n'avons pas divisé le territoire suivant des critères qui étaient valables il y a deux siècles, trois siècles, quatre siècles? Et est-ce que le temps n'est pas venu de redéfinir le territoire où responsabilités urbaines et responsabilités rurales se conjuguent? J'ai écrit toute une théorie là-dessus dans un livre que j'ai publié il y a 20 ans, Socialiste de raison ${ }^{3}$, et je m'y tiens.

F.P. : Vous l'évoquez en partie et, comme vous l'évoquez pour le passé...

Edgard Pisani : Vous êtes désespérés, mesdames et monsieur, vous êtes désespérés, mais il faut se battre (en tapant vigoureusement sur la table).

F.P. : Vous évoquez pour le passé l'effort important d'équipement du territoire qui a été fait dans l'immédiat après-guerre. En ce qui concerne la France et cette vitalisation rurale que vous évoquez ici, n'y a-t-il pas en même temps à conserver cet équipement du territoire, voire même à le renforcer, puisqu'on assiste au contraire avec les lois du marché à la disparition des services...

Edgard Pisani : Vous avez absolument raison.

F.P. : ... et aussi de tout ce qui a été fait en réseaux d'électricité, d'eau potable, etc.

\footnotetext{
${ }^{3}$ Pisani, E., 1978. Socialiste de raison : études et engagement politique, Paris, Flammarion.
} 
Edgard Pisani : Je vais prendre un exemple qui est très intéressant, je l'ai vécu, je parle d'expérience, madame Mathieu. Il y a en Haute-Marne 548 communes pour une population de l'ordre de 100000 habitants. J'en étais alors le préfet. Paris nous donnait les moyens de faire trois ou quatre adductions d'eau par an. C'est-à-dire qu'il me fallait 100 ans, ou quelque chose comme ça, pour qu'il y ait des adductions d'eau partout. J'ai réuni alors des maires, ici et là, j'ai réuni des assemblées populaires en posant la question : "Quand vous avez de l'eau au robinet, à la ferme pour les vaches, quel est le bénéfice que vous faites? » Réponses : «On ne va pas à la fontaine casser la glace» ; un autre : «Pendant le trajet, la vache perd bien un litre de lait, parce qu'il fait froid ».. « « Eh bien, grâce à un mètre cube d'eau, combien économisez-vous d'argent? - Disons 30, 20 litres de lait. - Et combien voulez-vous payer l'eau ? - Eh bien, 20 centimes, 30 centimes. » Je dis : «Quoi ? En ayant l'eau, vous gagnez 20 à 30 litres - disons 100 francs à l'époque -, et vous ne voulez payer que $1 \%$ ou $2 \%$ de ce que vous gagnez? Alors, nous allons faire une politique de l'eau chère. » C'est ainsi que cela s'est passé et vous pouvez aller fouiller dans les archives du département de Haute-Marne! « Vous allez accepter de payer l'eau chère et nous allons faire 5,10 fois plus d'adductions d'eau par an. » Je suis monté à Paris, j'ai négocié avec le ministère de l'Agriculture. Nous sommes allés à la caisse de Crédit agricole et à la Caisse des dépôts et consignations, on m'a prêté pour faire de la politique de l'eau. Entre-temps, j'ai été élu sénateur, j'ai fait ma campagne sur l'eau chère et j'ai été élu triomphalement. On nous raconte donc des sornettes sur les difficultés à faire une politique équitable! Vous pouvez aller en Haute-Marne, vous pouvez aller interroger les gens sur le terrain. Vous savez, en Haute-Marne, ce n'est pas la Bretagne où il y a de l'eau partout, on est sur un plateau calcaire où il faut trouver l'endroit d'où amener l'eau... Ainsi, en HauteMarne, pour en revenir à l'eau - j'ai envie de parler de l'eau -, j'ai fait faire, en 1954, le plan des ressources hydrauliques du département pour calquer le système des adductions d'eau sur le plan des ressources, et non pas sur les initiatives locales qui poussaient à pomper ici, à pomper là... Et le résultat, c'est qu'on est arrivé à avoir l'eau pour tout le monde. Et tout cela est possible, je vous assure que je ne rêve pas, je ne rêve pas, je l'ai fait. Sous prétexte qu'on a la légitimité qui résulte des élections, on méprise la réalité et on n'écoute les électeurs qu'aux marges, pour les séduire, mais on ne les écoute pas sur les problèmes fondamentaux. C'est cela le vrai problème de la démocratie.

Y.G. : En somme, pour faire de la politique, il faut lancer une perspective!

Edgard Pisani : Pour ce qui est des perspectives, hélas, j'en ai plein les poches!!!

\section{F.P. : C'est justement ça qui est extraordinaire!}

Edgard Pisani : Je vais vous parler d'une autre expérience... Vous m'emmerdez au fond! Je ne suis pas obligé de vous raconter tout cela!

\section{F.P. : Mais si ! On avait envie de vous embêter.}

Edgard Pisani : J'étais maire de Montreuil-Bellay où, comme je vous l'ai déjà dit, je ne vivais pas. J'ai décidé, après avis et délibération du conseil municipal, évidemment, de provoquer chaque année deux débats publics, et en particulier un avant le vote du budget, pour dire à la population ce que nous y mettions. Les rôles étaient répartis : celui des chemins parlait des chemins, celui des écoles parlait des écoles, celui des finances parlait du budget, et moi je ne disais rien. La fréquentation moyenne de ces réunions a été de l'ordre de 400 pour une population qui a dépassé 2500 pour aller à 3500 et 4000 . Il est arrivé une ou deux fois que des ivrognes viennent interrompre le débat : c'est la salle qui a dit aux gars de se taire et de foutre le camp. Le lendemain ou le surlendemain, je réunissais le conseil municipal. Eh bien, ce qui m'a frappé, c'est que le seul fait d'avoir à exposer un dossier devant des gens qui ne le connaissent pas vous apprend des choses que vous ne découvrez pas quand vous en parlez en technicien devant des techniciens. Le problème de la bureaucratie, c'est qu'elle se parle des dossiers à elle-même, sans se préoccuper de ceux auxquels ces dossiers s'imposent. Donc, le seul fait, par autoéducation, par automaturation si vous voulez, le seul fait de parler aux gens très simplement de telle sorte qu'ils comprennent bien ce qu'on leur dit - non pas le chapitre 22, paragraphe 3 , mais ce qui se joue concrètement - modifie l'action politique. J'en ai fait l'expérience directe la première fois que j'ai demandé : «Alors, quelle est la conclusion que vous tirez de ce débat? » Eh bien, plusieurs de mes adjoints qui avaient pris la parole $\mathrm{m}^{\prime}$ ont dit : «On ne peut pas faire ça ! » M'adressant à l'un d'entre eux en particulier, je lui demande : «Pourquoi dites-vous cela alors que personne n'a fait d'observation ? - Parce que j'ai senti qu'ils n'étaient pas d'accord. » Donc, nous avons une démocratie tronquée, une démocratie avec un fossé infranchissable entre l'élu et l'électeur. L'hypocrisie, le jeu de charme, voilà la réalité dans laquelle nous sommes. Enfin, mesdames, monsieur, pardonnez-moi, avec ma taille, avec ma voix, avec ma gueule, je passe pour un homme lointain. Et je suis un homme assez lointain, je ne me familiarise pas, si vous voulez... Mais j'écoute. Ils ne demandent pas qu'on leur tape sur le ventre!

F.P. : N'y a-t-il pas, néanmoins et en même temps, la même coupure que vous dénoncez entre l'expert et le politique? Vous avez des mots assez durs sur les experts qui n'en démordent pas lorsqu'ils ont écrit quelque chose. Par ailleurs, vous nous dites - et c'est un scoop quand même - que, lors de la négociation de la politique 
agricole commune, pour ce qui concerne le commerce alimentaire et les céréales, c'était bien, et vous l'avez découvert finalement plus tard, c'était bien la question de la concurrence Europe/États-Unis pour le marché international des produits alimentaires qui était en cause, et vraiment pas du tout une question de suffisance alimentaire de l'Europe qui naissait alors. Est-ce que cela veut dire que finalement...

Edgard Pisani : Je ne suis pas sûr que les experts fussent eux-mêmes tout à fait informés.

F.P. : Alors, justement, est-ce que les politiques et les experts, et les deux, se rendent réellement et toujours compte des effets induits - des boules de neige sur les territoires - de la politique qu'ils mettent en œuvre?

Edgard Pisani : Non! Non! Il faut faire des hypothèses, et parfois nous n'en faisons pas. Et nous avons tort. C'est parce que nous n'avons pas chez nous de cellules de prospective sur les conséquences de ce que nous faisons. Nous allons au doigt mouillé !

N.M. : La quatrième partie de votre livre s'intitule "Adresses aux responsables ", et on y trouve une allocution «à une assemblée de chercheurs ». Alors, puisque vous vous êtes intéressé aux chercheurs - même si ceux de l'Inra sont les principaux destinataires -, en essayant de trouver les mots mobilisateurs, le feriez-vous pour les géographes? Que leur diriez-vous?

Edgard Pisani : Ah, non! Pour les géographes, je ne suis pas sûr, parce que je ne suis pas compétent. Pour les éducateurs, peut-être.

N.M. : Mais vous pouvez imaginer, quand même?

Edgard Pisani : Madame, je suis devenu, par chance et peut-être par hasard, un géographe en ce sens que je connais beaucoup de monde, que j'ai vu beaucoup du monde. J'aurais tendance à tourner toute ma recherche autour de la dialogique unité-diversité. Il y a une identité des choses sur une extraordinaire diversité, une identité de problématiques fondamentales sur une extraordinaire diversité des conditions et des solutions des problèmes. Et donc, tout est jeu dialectique. Et la dialectique n'est jamais la même. Avec le même sentiment d'unité, vous ne raisonnez pas de la même façon devant deux éléments divers et différents. C'est-à-dire que rechercher l'unité - c'est ce que j'ai appelé médiation tout à l'heure - est une manière de faire que l'unité devienne la pierre angulaire du système, ce qui n'exclut pas que cette pierre angulaire tienne compte des différences. Parler d'unité de l'espèce humaine, parler d'unité du destin, donc de solidarité, ce n'est pas la même chose à Paris et à Ouagadougou. Cela ne peut pas être la même chose. Là est donc la base de toute une réflexion : intégrer la diversité, non pas comme un élément d'opposition, mais comme un élément d'enrichissement. Détruire le regard de touriste que nous enseignons à nos enfants quand nous leur parlons du monde! Pour apprendre! Nous sommes, les enfants sont des touristes partout, peut-être même chez eux. Il ne faut être touriste nulle part.

N.M. : Je m'inscris dans le prolongement de ce que vous venez de dire sur la géographie en cherchant à vous ramener au sujet qui nous occupe : la question de la nourriture et des agricultures. Le rapport entre géographie, agriculture et alimentation est, à nos yeux, essentiel. Et pourtant, ici à Paris 1 où j'ai enseigné la « rurale » pendant plus de trente ans, l'enseignement de géographie rurale et d'agriculture va disparaître, y compris sans doute dans le $3^{\mathrm{e}}$ cycle. Il me semble que cela rejoint ce que vous dites et nous éclaire sur les raisons pour lesquelles nous avons voulu vous faire venir : l'importance de maintenir, de renforcer ce lien fait entre la recherche, l'enseignement, etc., et ces questions fondamentales que sont l'agriculture et la nourriture. Que pouvez-vous nous dire pour nous encourager, encourager les géographes qui ont cette position?

Edgard Pisani : Avant de vous répondre - je vais le faire, bien sûr -, je voudrais poursuivre ma réflexion au point où elle en était. En fait, ma recherche est un vaet-vient permanent entre des termes apparemment alternatifs, disons trois couples de mots : c'est "unité et diversité », c'est "instant et durée » et c'est "ordre et désordre ». J'ai été un homme de l'État avant d'être un citoyen. J'ai été préfet à 27 ans... Je ne connaissais pas grand-chose... J'étais né en Tunisie... Donc, j'étais tout ordre dans ma tête. Et puis j'ai appris que l'ordre n'avait pas de sens sans son contraire, sans son partenaire, à la vérité, qui est le désordre. Et l'une des découvertes les plus bouleversantes de ma vie, c'est celle qu'ont faite les astronomes, les spécialistes de l'espace, le jour où ils ont découvert que l'espace n'obéissait pas à la loi qu'ils croyaient avoir édictée comme expliquant et faisant prévoir l'évolution des choses. Ainsi, ma recherche bascule, va constamment dans ces différents domaines, dans ces différentes approches. Je suis encore analphabète dans l'analyse de ce que je viens de dire, mais je suis certain que c'est là que se joue l'avenir. S'il me reste des années à vivre, c'est sans doute à cela que je consacrerai mon temps, ma réflexion, mes contacts, mes recherches, mes échanges...

Bon! Pour vous, madame [Nicole Mathieu], une anecdote - puisque, comme disait un homme qui me parlait de ma barbe : «Toi, tu parles par parabole. » J'ai envoyé mon livre à une des plus grandes personnalités industrielles de France qui dirige un laboratoire de réflexion. Il m'a envoyé une lettre : «Monsieur, vous m'avez fait découvrir que, dans toutes les stratégies que nous avons étudiées, nous avons oublié un facteur essentiel qui est 
celui de l'agriculture, de l'alimentation et de l'environnement. » Il me demande de faire partie d'un groupe d'études qu'il va créer pour essayer de voir comment assurer l'imbrication réciproque du contenu de mon livre et du reste ! C'est une anecdote qui pèse lourd. $\mathrm{Sij}$ javais à dire ce que j'ai appris au cours de cette année ou presque - ces huit ou neuf mois de travail autour de mon livre-, il me suffirait d'une phrase, et pourtant la moisson a été très riche. Alors quej'ai fait mon livre en plaidant pour l'agriculture, aujourd'hui, si je le réécrivais, je plaiderais pour un monde non agricole, qui serait privé de l'agriculture $q^{\prime}{ }^{\prime} i l$ est en train de détruire. C'est le monde non agricole qui a besoin de l'agriculture, ce n'est pas l'agriculture. À cause des subventions, à cause des lois, à cause de tout le bazar, on a le sentiment que les agriculteurs sont des mendiants, qu'ils quêtent leur subsistance. Or, à la vérité c'est demain, peut-être déjà aujourd'hui, que le monde non agricole est en état de demandeur à l'égard de l'agriculture, ceci pour des raisons d'alimentation - la double sécurité alimentaire -, d'environnement et d'espace, et de société rurale. Pour ce qui est des sociétés rurales, on peut prendre cet exemple qui est plus facile à expliquer. Il y a actuellement 800 millions de ruraux en Chine. L'année dernière, 25 millions ont été installés dans les villes. Alors, première question : Combien coûte l'installation d'un poilu dans une ville? Minimum 10000 euros. 25 millions par 10000 euros, ça fait beaucoup, beaucoup d'argent. Le transfert du monde rural dans le monde urbain pose un problème d'investissement tout à fait redoutable. Le deuxième problème est : Que vont-ils faire en ville? Sur les 25 millions, il y en aura 10 ou 9 millions, peu importe, qui vont devenir ouvriers. Qu'est-ce qu'ils vont faire? Des automobiles, des ordinateurs, du textile... Et un jour, c'est nous qui fermerons nos marchés. Parlons enfin de l'environnement. Il est clair que la détérioration de l'environnement provient du cumul de trois phénomènes qui ne sont pas de nature comparable : des phénomènes cycliques, des phénomènes qui résultent de la pollution industrielle, et puis ceux qui résultent de l'usage du sol. Quelques exemples du rythme où vont les choses : l'urbanisation en France détruit de l'ordre de 5000 hectares de terres utiles par an, peut-être un peu moins maintenant; il y a 1200000 hectares de désert qui se crée chaque année dans le monde, peut-être un peu plus. Dans la logique de notre évolution, nous sommes en train de détruire la terre sur laquelle nous vivons. Que ce soit en termes de sécurité alimentaire, que ce soit en termes de sociétés rurales, que ce soit en termes d'environnement, c'est maintenant le monde non agricole qui doit s'interroger sur son avenir dans un monde dont il aurait détruit l'agriculture. J'ai inversé complètement ma logique. C'est long d'inverser une logique, parce qu'il faut tout reprendre en sens inverse pour dire la même chose, finalement. C'est actuellement la leçon que je tire de cette année d'expérience.
N.M. : Du coup, vous me donnez le courage d'exprimer ce que je pense : nous devons devenir tous des paysans. Vous, vous êtes dans une réflexion fondamentale, dans une sorte de révolution, du côté de l'action politique, du Politique au sens collectif. Et moi, je reprends ce que vous dites comme un individu, comme un habitant ordinaire... Car cette révolution mentale, cette même capacité critique de renversement, de reformulation du politique, est quelque chose qui doit aussi toucher chacun d'entre nous. Chaque personne doit faire ce travail-là, ne serait-ce que se demander ce que veut dire se nourrir.

Edgard Pisani : J'ai dirigé une revue qui s'appelait L'Événement européen, et qui est morte parce que les partis socialistes européens ne voulaient pas se mettre d'accord entre eux. Mais ce n'est pas là l'objet. Elle est morte aussi parce que je me suis séparé - je dirais idéologiquement, mais pas personnellement parce que nous sommes restés copains - de quelqu'un qui travaillait avec moi dans cette revue. Cet homme m'a dit un jour : "Je ne me sens de nulle part, quand je dis que je suis méditerranéen, c'est du milieu de la Méditerranée. » Je lui ai posé la question : «Du milieu de l'eau, là ? - Oui, du milieu de l'eau. » Je l'ai regardé, je lui ai dit : "Mais moi non. Je suis méditerranéen parce que j'ai les pieds sur la terre, sur une terre autour de la Méditerranée. » Alors, tout ça pour dire qu'au-delà du concept que je vais aborder maintenant, il y a un autre phénomène qui me paraît se développer et prendre une place extrêmement importante et extrêmement grave, c'est la déterritorialisation. On se déculture, on se désappartient. On se veut comme un ludion se promenant partout, n'appartenant à personne, et Internet n'y est pas pour rien. On est de partout et donc de nulle part. Eh bien, ma réponse à moi est : «Je suis de quelque part pour être de partout. » $C^{\prime}$ est donc une philosophie absolument opposée. À partir de là, j'en arrive à la question que vous m'avez posée. Madame, il faut que chacun mesure, il faudrait que chacun mesure profondément qu'il est paysan parce qu'il dépend du pays, c'est-à-dire au fond de la terre, qui en même temps appartient à tout le monde et n'appartient à personne. La terre appartient à tout le monde. C'est cette dialectique, toute la terre nous appartient et, si nous en possédons une parcelle, ce n'est qu'une part, comme usufruitier. Et donc, la valorisation du terme de paysan, ce que je tente en quelques phrases dans mon livre, est une véritable anxiété chez moi. Redevenir, se ressentir de quelque part... C'est en effet un problème beaucoup plus large. Ce qu'on peut dire aussi, c'est que la mondialisation, c'est aussi la déterritorialisation de la production. Déterritorialisation de la production puisqu'on peut acheter n'importe où n'importe quoi, ce qui conduit progressivement au désenracinement des êtres. Et donc, je vais vous dire ma conclusion générale. Je n'ai jamais parlé autant 
de moi-même et des choses auxquelles je crois dans ma vie. Merci de m'en avoir donné l'occasion. Je traverse un moment très crucial dans ma propre évolution. Au fond, je crois que nous vivons le Moyen Âge, c'est-à-dire un moment où tout est mis en cause et où tous les éléments à partir desquels nous entendons juger des choses se multiplient. Et nous n'avons pas encore esquissé l'ordre dans lequel nous voulons rebâtir le monde. Alors, entendonsnous bien, monsieur, je comprends. Le mot ordre, dans le dictionnaire philosophique de Lalande, c'est un aménagement intelligible des choses. Nous avons cru jusque dans les années 1940-1950, voire même 1960, vivre dans un monde intelligible, et nous avons maintenant le sentiment que nous vivons dans un monde inintelligible. Alors, la question est posée et elle est essentielle. Moi, je ne peux pas répondre à la question de savoir si on peut vivre dans un monde inintelligible, puisque je ne sais pas. Je pars donc de l'hypothèse que nous avons besoin d'un monde intelligible. En cela, mon raisonnement pèche d'une certaine façon puisque j'exclus une des deux hypothèses. Mais quel peut être le monde intelligible vers lequel nous allons? Et ça, je ne le sais pas encore. Je ne le saurai jamais.
Entretien avec Edgard Pisani réalisé, le 17 septembre 2004 dans la bibliothèque de l'Institut de géographie (191 rue Saint-Jacques, à Paris), par Nicole Mathieu, Françoise Plet et Yves Guermond, à l'occasion de la sortie de son livre Un vieil homme et la terre : neuf milliards d'êtres à nourrir, la nature et les sociétés rurales à sauvegarder (Le Seuil, 2004) ${ }^{a}$. L'entretien, filmé intégralement ( 1 h $44 \mathrm{~min} 46 \mathrm{~s}$ ) par Olivier rchambeau, docteur en géographie et chargé de conférences à l'Université de Paris 8, a été retranscrit par Nicole Mathieu. Un montage vidéo (22 min 27 s), réalisé par Françoise Plet et Philippe Gajewski, en a été extrait : Edgard Pisani. Produire pour nourrir, ou l'Écoute et l'intelligence des territoires; il a été présenté au nom de l'Association française pour le développement de la géographie (AFDG) au $15^{\mathrm{e}}$ Festival international de géographie en 2004 à Saint-Dié-des-Vosges Nourrir les hommes, nourrir le monde. Les géographes se mettent à table -, dans une conférence-débat avec Nicole Mathieu, Françoise Plet et Noël Copin. (Il peut être visionné sur Internet, à l'url : http://www.afdg.org/spip/video/index.htm) Natures Sciences Sociétés publie les extraits de l'entretien qui mettent en valeur le rapport de cet homme politique, ayant exercé de nombreuses et importantes responsabilités d'État, avec la connaissance et l'expérience.

a Voir dans la rubrique Ouvrages en débat de ce numéro, le compte rendu de cet ouvrage rédigé par B. Hubert. 